\title{
Towards fault-tolerant quantum computing with trapped ions
}

\author{
JAN BENHELM, GERHARD KIRCHMAIR, CHRISTIAN F. ROOS* AND RAINER BLATT \\ Institut für Experimentalphysik, Universität Innsbruck, Technikerstr. 25, A-6020 Innsbruck, Austria \\ Institut für Quantenoptik und Quanteninformation, Österreichische Akademie der Wissenschaften, Otto-Hittmair-Platz 1, A-6020 Innsbruck, Austria \\ *e-mail: Christian.Roos@uibk.ac.at
}

Today, ion traps are among the most promising physical systems for constructing a quantum device harnessing the computing power inherent in the laws of quantum physics, ${ }^{1,2}$. For the implementation of arbitrary operations, a quantum computer requires a universal set of quantum logic gates. As in classical models of computation, quantum error correction techniques ${ }^{3,4}$ enable rectification of small imperfections in gate operations, thus enabling perfect computation in the presence of noise. For fault-tolerant computation ${ }^{5}$, it is believed that error thresholds ranging between $10^{-4}$ and $10^{-2}$ will be required-depending on the noise model and the computational overhead for realizing the quantum gates $^{6-8}$ - but so far all experimental implementations have fallen short of these requirements. Here, we report on a Mølmer-Sørensen-type gate operation ${ }^{9,10}$ entangling ions with a fidelity of $99.3(1) \%$. The gate is carried out on a pair of qubits encoded in two trapped calcium ions using an amplitudemodulated laser beam interacting with both ions at the same time. A robust gate operation, mapping separable states onto maximally entangled states is achieved by adiabatically switching the laser-ion coupling on and off. We analyse the performance of a single gate and concatenations of up to 21 gate operations.

For ion traps, all building blocks necessary for the construction of a universal quantum computer ${ }^{1}$ have been demonstrated over the past decade. Currently, the most important challenges consist of scaling up the present systems to a higher number of qubits and raising the fidelity of gate operations up to the point where quantum error correction techniques can be successfully applied. Although single-qubit gates are easily carried out with high quality, the realization of high-fidelity entangling two-qubit gates ${ }^{11-16}$ is much more demanding because the inter-ion distance is orders of magnitude bigger than the characteristic length scale of any state-dependent ion-ion interaction. Apart from quantum gates of the Cirac-Zoller type ${ }^{2,12}$, where a laser couples a single qubit with a vibrational mode of the ion string at a time, most other gate realizations entangling ions have relied on collective interactions of the qubits with the laser control fields ${ }^{11,13-15}$. These gate operations entangle transiently the collective pseudospin of the qubits with the vibrational mode and produce either a conditional phase shift ${ }^{17}$ or a collective spin flip ${ }^{9,10,18}$ of the qubits. Whereas the highest fidelity $F=97 \%$ reported until now ${ }^{13}$ has been achieved with a conditional phase gate acting on a pair of hyperfine qubits in ${ }^{9} \mathrm{Be}^{+}$, spin-flip gates have been limited so far to $F \approx 85 \%$ (refs 11,14). All of these experiments have used qubits encoded in hyperfine or Zeeman ground states and a Raman transition mediated by an electric-dipole transition for coupling the qubits.

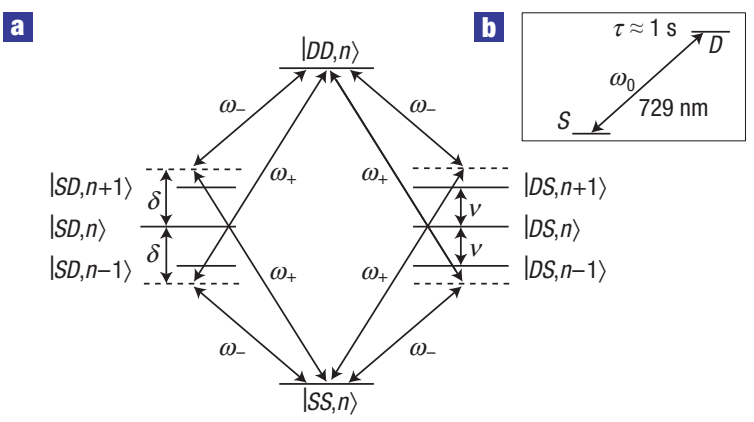

Figure 1 Gate mechanism. a, A bichromatic laser field with frequencies $\omega_{+}, \omega_{-}$ satisfying $2 \omega_{0}=\omega_{+}+\omega_{-}$is tuned close to the upper and lower motional sideband of the qubit transition. The field couples the qubit states $|S S\rangle \leftrightarrow|D D\rangle$ through the four interfering paths shown in the figure, $n$ denoting the vibrational quantum number of the axial COM mode. Similar processes couple the states $|S D\rangle \leftrightarrow|D S\rangle$. b. The qubits are encoded in the ground state $S_{1 / 2}(m=1 / 2)$ and the metastable state $D_{5 / 2}(m=3 / 2)$ of ${ }^{40} \mathrm{Ca}^{+}$ions and are manipulated by a narrow bandwidth laser emitting at a wavelength of $729 \mathrm{~nm}$.

Whereas spontaneous scattering from the mediating short-lived levels degrades the gate fidelity owing to the limited amount of laser power available in current experiments ${ }^{19}$, this source of decoherence does not occur for optical qubits, that is, qubits encoded in a ground state and a metastable electronic state of an ion. In the experiment presented here, where the qubit comprises the states $|S\rangle \equiv S_{1 / 2}(m=1 / 2)$ and $|D\rangle \equiv D_{5 / 2}(m=3 / 2)$ of the isotope ${ }^{40} \mathrm{Ca}^{+}$, spontaneous decay of the metastable state reduces the gate fidelity by less than $5 \times 10^{-5}$.

A Mølmer-Sørensen gate inducing collective spin flips is achieved with a bichromatic laser field with frequencies $\omega_{ \pm}=\omega_{0} \pm \delta$, with $\omega_{0}$ being the qubit transition frequency and $\delta$ close to the vibrational mode frequency $v$ (Fig. 1). For optical qubits, the bichromatic field can be a pair of co-propagating lasers, which is equivalent to a single laser beam resonant with the qubit transition and amplitude-modulated with frequency $\delta$. For a gate mediated by the axial centre-of-mass (COM) mode, the hamiltonian describing the laser-qubit interaction is given by $H=\hbar \Omega \mathrm{e}^{-i \phi} S_{+}\left(\mathrm{e}^{-i(\delta t+\zeta)}+\mathrm{e}^{i(\delta t+\zeta)}\right) \mathrm{e}^{i \eta\left(a \mathrm{e}^{-i v t}+a^{\dagger} \mathrm{e}^{i v t}\right)}+$ h.c. Here, $S_{j}=\sigma_{j}^{(1)}+\sigma_{j}^{(2)}, j \in\{+,-, x, y, z\}$, denotes a collective atomic operator constructed from Pauli spin operators $\sigma_{j}^{(i)}$ acting on ion 

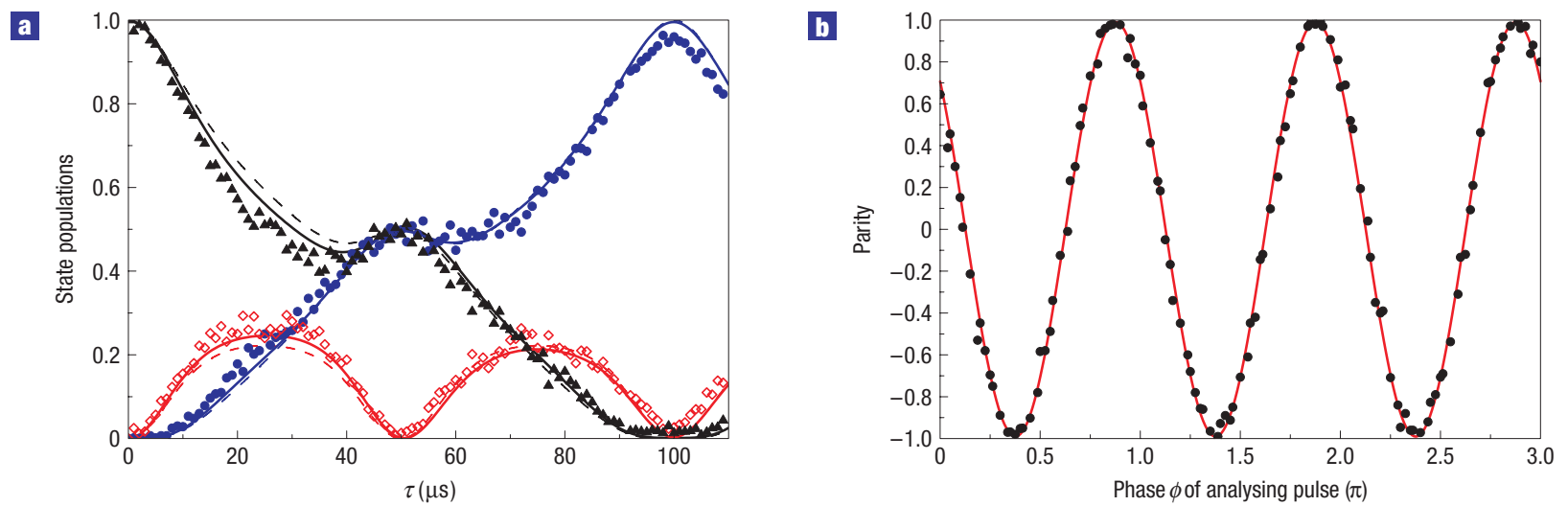

Figure 2 High-fidelity gate operation. a, Evolution of the populations $p_{0}$ (filled circle), $p_{1}$ (open diamond) and $p_{2}$ (filled triangle) induced by a Mølmer-Sørensen bichromatic pulse of duration $\tau$. The Rabi frequency $\Omega(t)$ is smoothly switched on and off within $2 \mu \mathrm{s}$ and adjusted such that a maximally entangled state is created at $\tau_{\text {gate }}=50 \mu \mathrm{s}$. The dashed lines are calculated for $\bar{n}_{\mathrm{COM}}=0.05$ from the propagator (1), neglecting pulse shaping and non-resonant carrier excitation. The solid lines are obtained by numerically solving the Schrödinger equation for time-dependent $\Omega(t)$ and imbalanced Rabi frequencies $\Omega_{+} / \Omega_{-}=1.094$ (see the Methods section). $\mathbf{b}, \mathrm{A}(\pi / 2)_{\phi}$ analysis pulse applied to both ions prepared in $\Psi_{1}$ gives rise to a parity oscillation $P(\phi)=\sin (2 \phi)$ as a function of $\phi$. A fit with a function $P_{\text {fit }}=A \sin \left(2 \phi+\phi_{0}\right)$ yields the parity fringe amplitude $A=0.990(1)$ and $\phi_{0} / \pi=-1.253(1)$. The precise value of the phase $\phi_{0}$ is without significance. It arises from phase-locking the frequencies $\omega_{0}, \omega_{+}, \omega_{-}$and could have been experimentally adjusted to zero.

$i$, and $\sigma_{+}^{(i)}|S\rangle_{i}=|D\rangle_{i}$. The operators $a, a^{\dagger}$ annihilate and create phonons of the COM mode with Lamb-Dicke factor $\eta$. The optical phase of the laser field (with coupling strength $\Omega$ ) is labelled $\phi$, and the phase $\zeta$ accounts for a time difference between the start of the gate operation and the maximum of the amplitude modulation of the laser beam. In the Lamb-Dicke regime, and for $\phi=0$, the gate operation is very well described by the propagator ${ }^{20}$

$$
U(t)=\mathrm{e}^{-i F(t) S_{x}} \hat{D}\left(\alpha(t) S_{y, \psi}\right) \exp \left(-i(\lambda t+\chi \sin (\nu-\delta) t) S_{y, \psi}^{2}\right) .
$$

Here, the operator to the right describes collective spin flips induced by the operator $S_{y, \psi}=S_{y} \cos \psi+S_{z} \sin \psi$, $\psi=(4 \Omega / \delta) \cos \zeta$, and $\lambda \approx \eta^{2} \Omega^{2} /(v-\delta), \chi \approx \eta^{2} \Omega^{2} /(v-\delta)^{2}$. With $\alpha(t)=\alpha_{0}\left(\mathrm{e}^{i(\nu-\delta) t}-1\right)$, the displacement operator $\hat{D}(\beta)=\mathrm{e}^{\beta a^{\dagger}-\beta^{*} a}$ accounting for the transient entanglement between the qubits and the harmonic oscillator becomes equal to the identity after the gate time $\tau_{\text {gate }}=2 \pi /|\nu-\delta|$. The operator $\mathrm{e}^{-i F(t) S_{x}}$ with $F(t)=(2 \Omega / \delta)(\sin (\delta t+\phi)-\sin \phi)$ describes fast non-resonant excitations of the carrier transition that occur in the limit of short gates when $\Omega \ll \delta$ no longer strictly holds. Non-resonant excitations are suppressed by intensity-shaping the laser pulse so that the Rabi frequency $\Omega(t)$ is switched on and off smoothly. Moreover, adiabatic switching makes the collective spin-flip operator independent of $\zeta$ as $S_{y, \psi} \rightarrow S_{y}$ for $\Omega \rightarrow 0$. To achieve adiabatic following, it turns out to be sufficient to switch on the laser within 2.5 oscillation periods of the ions' axial COM mode. When the laser is switched on adiabatically, equation (1) can be simplified by dropping the factor $\mathrm{e}^{-i F(t) S_{x}}$ and replacing $S_{y, \psi}$ by $S_{y}$. To realize an entangling gate of duration $\tau_{\text {gate }}$ described by the unitary operator $U_{\text {gate }}=\exp \left(-i(\pi / 8) S_{y}^{2}\right)$, the laser intensity needs to be set such that $\eta \Omega \approx|\delta-\nu| / 4$.

Two ${ }^{40} \mathrm{Ca}^{+}$ions are confined in a linear trap ${ }^{21}$ with axial and radial COM mode frequencies of $v_{\text {axial }} / 2 \pi=1.23 \mathrm{MHz}$ and $\nu_{\text {radial }} / 2 \pi=4 \mathrm{MHz}$, respectively. After Doppler cooling and frequency-resolved optical pumping ${ }^{22}$ in a magnetic field of $4 \mathrm{G}$, the two axial modes are cooled close to the motional ground state $\left(\bar{n}_{\mathrm{COM}}, \bar{n}_{\text {stretch }}<0.05(5)\right)$. Both ions are now initialized to $|S S\rangle$ with a probability of more than $99.8 \%$. Then, the gate operation is carried out, followed by an optional carrier pulse for analysis. Finally, we measure the probability $p_{k}$ of finding $k$ ions in the $|S\rangle$ state by detecting light scattered on the $S_{1 / 2} \leftrightarrow P_{1 / 2}$ dipole transition with a photomultiplier for $3 \mathrm{~ms}$. The error in state detection due to spontaneous decay from the $D$ state is estimated to be less than $0.15 \%$. Each experimental cycle is synchronized with the frequency of the a.c.-power line and repeated 50-200 times. The laser beam carrying out the entangling operation is controlled by a double-pass acousto-optic modulator, which enables setting the frequency $\omega_{\mathrm{L}}$ and phase $\phi$ of the beam. By means of a variable gain amplifier, we control the radiofrequency input power and hence the intensity profile of each laser pulse. To generate a bichromatic light field, the beam is passed through another acousto-optic modulator in single-pass configuration that is driven simultaneously by two radiofrequency signals with difference frequency $\delta / \pi$ (see the first paragraph of the Methods section). Phase coherence of the laser frequencies is maintained by phase-locking all radiofrequency sources to an ultrastable quartz oscillator. We use $1.8 \mathrm{~mW}$ average light power focused down to a spot size of $14 \mu \mathrm{m}$ gaussian beam waist illuminating both ions from an angle of $45^{\circ}$ with equal intensity to achieve the Rabi frequencies $\Omega /(2 \pi) \approx 110 \mathrm{kHz}$ required for carrying out a gate operation with $(\nu-\delta) /(2 \pi)=20 \mathrm{kHz}$ and $\eta=0.044$. To make the bichromatic laser pulses independent of the phase $\zeta$, the pulse is switched on and off by using Blackman-shaped pulse slopes of duration $\tau_{r}=2 \mu \mathrm{s}$.

Multiple application of the bichromatic pulse of duration $\tau_{\text {gate }}$ ideally maps the state $|S S\rangle$ to

$$
\begin{gathered}
|S S\rangle \stackrel{\tau_{\text {gate }}}{\longrightarrow} \underbrace{|S S\rangle+i|D D\rangle}_{\Psi_{1}} \stackrel{\tau_{\text {gate }}}{\longrightarrow}|D D\rangle \stackrel{\tau_{\text {gate }}}{\longrightarrow}|D D\rangle \\
+i|S S\rangle \stackrel{\tau_{\text {gate }}}{\longrightarrow}|S S\rangle \stackrel{\tau_{\text {gate }}}{\longrightarrow} \cdots
\end{gathered}
$$

up to global phases. Maximally entangled states occur at instances $\tau_{m}=m \cdot \tau_{\text {gate }}(m=1,3, \ldots)$. A similar mapping of product states onto Bell states and vice versa also occurs when starting from state $|S D\rangle$. To assess the fidelity of the gate operation, we adapt the strategy first applied in refs 11,13 consisting of measuring the fidelity of Bell states created by a single application of the gate to the state $|S S\rangle$ (Fig. 2a). The fidelity 


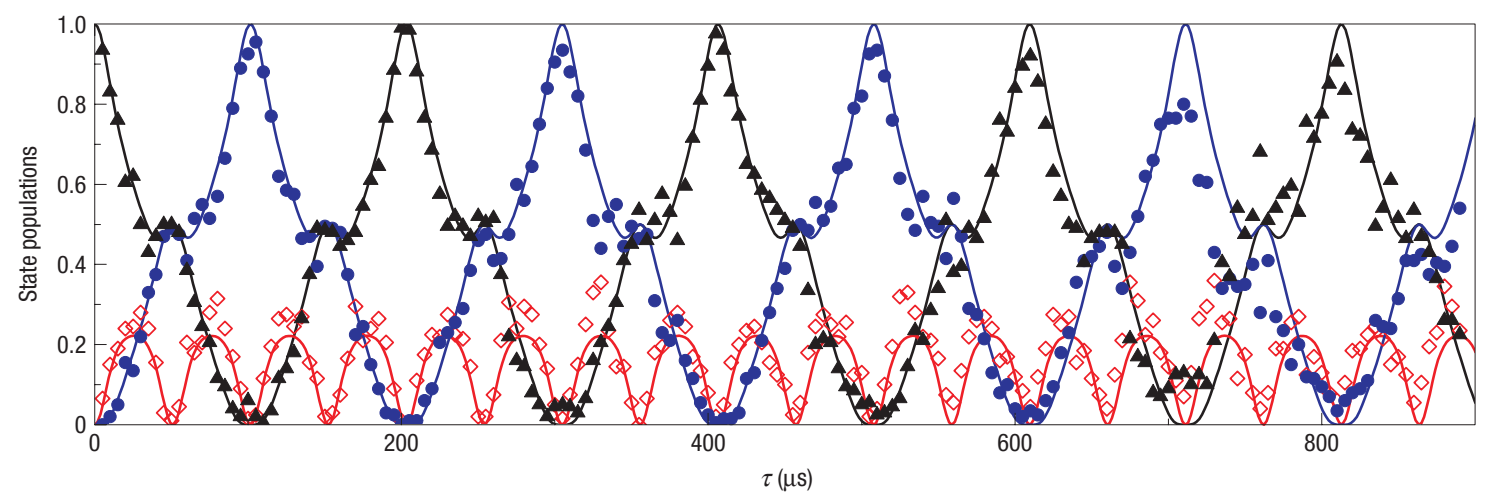

Figure 3 Entanglement and disentanglement dynamics of the Mølmer-Sørensen interaction. Starting from state $|S S\rangle$ for a detuning of the bichromatic laser from the sidebands set to $\delta-v=-20 \mathrm{kHz}$, the figure shows the time evolution of the populations $p_{0}, p_{1}$ and $p_{2}$ denoted by the filled circles, open diamonds and filled triangles respectively. The length of the pulse is equivalent to the application of up to 17 gate operations. Maximally entangled states are created whenever $p_{0}(\tau)$ and $p_{2}(\tau)$ coincide and $p_{1}(\tau)$ vanishes.

$F=\left\langle\Psi_{1}\left|\rho^{\exp }\right| \Psi_{1}\right\rangle=\left(\rho_{S S, S S}^{\exp }+\rho_{D D, D D}^{\exp }\right) / 2+\operatorname{Im} \rho_{D D, S S}^{\exp }$, with the density matrix $\rho^{\text {exp }}$ describing the experimentally produced qubits' state, is inferred from measurements on a set of 42,400 Bell states continuously produced within a measurement time of $35 \mathrm{~min}$. Fluorescence measurements on 13,000 Bell states reveal that $\rho_{S S, S S}^{\exp }+\rho_{D D, D D}^{\exp }=p_{2}+p_{0}=0.9965(4)$. The off-diagonal element $\rho_{D D, S S}^{\exp }$ is determined by measuring $P(\phi)=\left\langle\sigma_{\phi}^{(1)} \sigma_{\phi}^{(2)}\right\rangle$ for different values of $\phi$, where $\sigma_{\phi}=\sigma_{x} \cos \phi+\sigma_{y} \sin \phi$, by applying $(\pi / 2)_{\phi}$ pulses to the remaining 29,400 states and measuring $p_{0}+p_{2}-p_{1}$ to obtain the parity $\left\langle\sigma_{z}^{(1)} \sigma_{z}^{(2)}\right\rangle$. The resulting parity oscillation $P(\phi)$ shown in Fig. $2 \mathrm{~b}$ is fitted with a function $P_{\text {fit }}(\phi)=A \sin \left(2 \phi+\phi_{0}\right)$ that yields $A=2\left|\rho_{D D, S S}^{\exp }\right|=0.990(1)$. Combining the two measurements, we obtain the fidelity $F=99.3(1) \%$ for the Bell state $\Psi_{1}$.

A wealth of further information is obtained by studying the state dynamics under the action of the gate hamiltonian (see equation (2)). Starting from state $|S S\rangle$, Fig. 3 shows the time evolution of the state populations for pulse lengths equivalent to up to 17 gate times. The ions are entangled and disentangled consecutively up to nine times, the populations closely following the predicted unitary evolution of the propagator (1) for $\zeta=0$ shown in Fig. 3 as solid lines.

To study sources of gate imperfections we measured the fidelity of Bell states obtained after a pulse length $\tau_{m}$ for up to $m=21$ gate operations. The sum of the populations $p_{0}(t)+p_{2}(t)$ does not return perfectly to one at times $\tau_{m}$ as shown in Fig. 4 but decreases by about $0.0022(1)$ per gate. This linear decrease could be explained by resonant spin-flip processes caused by spectral components of the qubit laser that are far outside the laser's linewidth of $20 \mathrm{~Hz}$ (ref. 21) (see the Methods section). The figure also shows the amplitude of parity fringe pattern scans at odd integer multiples of $\tau_{\text {gate }}$ similar to the one in Fig. $2 \mathrm{~b}$. The gaussian shape of the amplitude decay is consistent with variations in the coupling strength $\Omega$ that occur from one experiment to the next (see the Methods section).

The observed Bell-state infidelity of $7 \times 10^{-3}$ indicates that the gate operation has an infidelity below the error threshold required by some models of fault-tolerant quantum computation $^{6-8}$ (an indication to be confirmed by full quantum gate tomography ${ }^{16}$ in future experiments). However, further experimental advances will be needed before fault-tolerant computation will become a reality as the overhead implied by these models is considerable. Nevertheless, in addition to making the implementation of

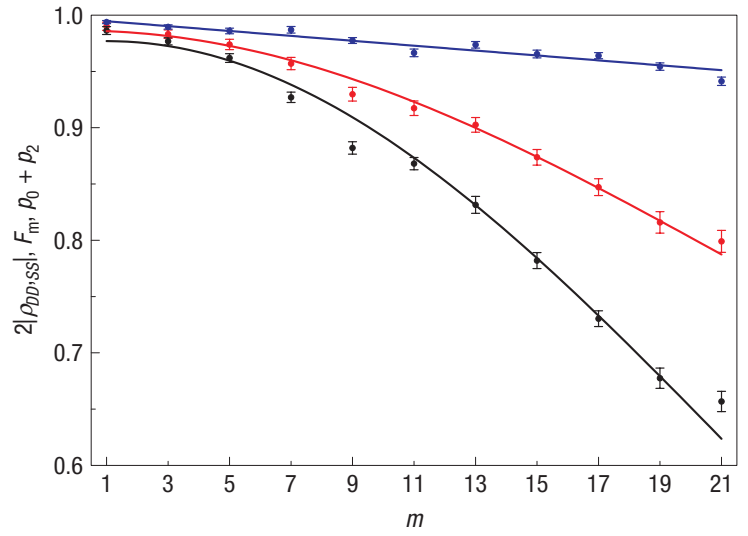

Figure 4 Multiple gate operations. Gate imperfections as a function of the bichromatic pulse length $\tau_{m}=m \cdot \tau_{\text {gate }}$ given in equivalent number of gate operations $m$. The upper curve shows a linear decrease of the state populations $p_{0}+p_{2}$ with a slope of $0.0022(1)$. All errors given are $1 \sigma$ statistical errors. The lower curve represents the magnitude of the coherence $2 \rho_{D D, S S}$ measured by detecting parity oscillations and fitted by a gaussian decay function that accounts for low-frequency noise of the laser-ion coupling strength $\Omega$ (see the Methods section). Combining both measurements yields the Bell-state fidelity $F_{m}$ shown as the middle trace. For $m=21$, the fidelity is still $F_{21}=80(1) \%$. Similar results are achieved when replacing the entangling pulse of length $\tau_{m}$ by $m$ amplitude-shaped pulses each of which is realizing an entangling gate operation.

quantum algorithms with tens of entangling operations look realistic, the gate presented here also opens interesting perspectives for generating multiparticle entanglement ${ }^{23}$ by a single laser interacting with more than two qubits at once. For the generation of $N$-qubit Greenberger-Horne-Zeilinger states, there exist no constraints on the positioning of ions in the bichromatic beam that otherwise made generation of Greenberger-Horne-Zeilinger states beyond $N=6$ difficult in the experiment with hyperfine qubits described in ref. 24. Although the bichromatic force lacks a strong spatial modulation that would enable tailoring of the gate interaction by choosing particular ion spacings ${ }^{25,26}$, more complex multiqubit interactions could be engineered by interleaving entangling laser pulses addressing all qubits with 
a focused laser inducing phase shifts in single qubits. Akin to nuclear magnetic resonance techniques, this method should enable refocusing of unwanted qubit-qubit interactions ${ }^{27}$ and open the door to a wide variety of entangling multiqubit interactions.

\section{METHODS}

\section{A.C.-STARK-SHIFT COMPENSATION}

The red-and the blue-detuned frequency components $\omega_{ \pm}$of the bichromatic light field cause dynamic (a.c.-) Stark shifts by non-resonant excitation on the carrier and the first-order sidebands that exactly cancel each other if the corresponding laser intensities $I_{ \pm}$are equal. The remaining a.c.-Stark shift due to other Zeeman transitions and far-detuned dipole transitions amounts to $7 \mathrm{kHz}$ for a gate time $\tau_{\text {gate }}=50 \mu$ s. These shifts could be compensated by using an extra far-detuned light field ${ }^{28}$ or by properly setting the intensity ratio $I_{+} / I_{-}$. We use the latter technique, which makes the coupling strengths $\Omega_{S S \leftrightarrow D D} \propto 2 \sqrt{I_{+} I_{-}}, \Omega_{S D \leftrightarrow D S} \propto I_{+}+I_{-}$slightly unequal. However, the error is insignificant as $\Omega_{S D \leftrightarrow D S} / \Omega_{S S \leftrightarrow D D}-1=4 \times 10^{-3}$ in our experiments.

\section{SOURCES OF GATE INFIDELITY}

A bichromatic force with time-dependent $\Omega(t)$ acting on ions prepared in an eigenstate of $S_{y}$ creates coherent states $\alpha(t)$ following trajectories in phase space that generally do not close $e^{20,29}$. For the short rise times used in our experiments, this effect can be made negligibly $\left(<10^{-4}\right)$ small by slightly increasing the gate time.

Spin flips induced by incoherent off-resonant light of the bichromatic laser field reduce the gate fidelity. A beat frequency measurement between the gate laser and a similar independent laser system that was spectrally filtered indicates that a fraction $\gamma$ of about $2 \times 10^{-7}$ of the total laser power is contained in a $20 \mathrm{kHz}$ bandwidth $B$ around the carrier transition when the laser is tuned close to a motional sideband. A simple model predicts spin flips to cause a gate error with probability $p_{\text {flip }}=(\pi \gamma|v-\delta|) /\left(2 \eta^{2} B\right)$. This would correspond to a probability $p_{\text {flip }}=8 \times 10^{-4}$, whereas the measured state populations shown in Fig. 4 would be consistent with $p_{\text {flip }}=2 \times 10^{-3}$. Spin-flip errors could be further reduced by two orders of magnitude by spectrally filtering the laser light and increasing the trap frequency $\nu /(2 \pi)$ to above $2 \mathrm{MHz}$ where noise caused by the laser frequency stabilization is much reduced.

Imperfections due to low-frequency noise randomly shifting the laser frequency $\omega_{\mathrm{L}}$ with respect to the atomic transition frequency $\omega_{0}$ were estimated from Ramsey measurements on a single ion showing that an average frequency deviation $\sigma_{\left(\omega_{\mathrm{L}}-\omega_{0}\right)} /(2 \pi)=160 \mathrm{~Hz}$ occurred. From numerical simulations, we infer that for a single gate operation this frequency uncertainty gives rise to a fidelity loss of $0.25 \%$ (an infidelity of $10^{-4}$ would require $\left.\sigma_{\left(\omega_{\mathrm{L}}-\omega_{0}\right)} /(2 \pi)=30 \mathrm{~Hz}\right)$. In our parity oscillation experiments shown in Figs $2 \mathrm{~b}$ and 4 , however, this loss is not directly observable because a small error in the frequency of the bichromatic laser beam carrying out the gate operation is correlated with a similar frequency error of the carrier $\left(\frac{\pi}{2}\right)_{\phi}$ pulse probing the entanglement produced by the gate so that the phase $\phi$ of the analysing pulse with respect to the qubit state remains well defined.

Variations in the coupling strength $\delta \Omega$ induced by low-frequency laser intensity noise and thermally occupied radial modes were inferred from an independent measurement by recording the amplitude decay of carrier oscillations. Assuming a gaussian decay, we find a relative variation of $\delta \Omega / \Omega=1.4(1) \times 10^{-2}$. For $m$ entangling gate operations, the loss of fidelity is approximately given by $1-F=(\pi m / 2)^{2}(\delta \Omega / \Omega)^{2}$ and contributes with
$5 \times 10^{-4}$ to the error of a single gate operation. For the multiple gate operations shown in Fig. 4, this source of noise explains the gaussian decay of the parity fringe amplitude, whereas laser frequency noise reduces the fringe amplitude by less than $1 \%$ even for 21 gate operations. In combination with error estimates for state preparation, detection and laser noise, the analysis of multiple gates provides us with a good understanding of the most important sources of gate infidelity.

Received 19 December 2007; accepted 25 March 2008; published 27 April 2008.

\section{References}

1. Nielsen, M. A. \& Chuang, I. L. Quantum Computation and Quantum Information (Cambridge Univ. Press, Cambridge, 2000).

2. Cirac, J. I. \& Zoller, P. Quantum computations with cold trapped ions. Phys. Rev. Lett. 74, 4091-4094 (1995).

3. Shor, P. W. Scheme for reducing decoherence in quantum computer memory. Phys. Rev. A 52, R2493-R2496 (1995)

4. Steane, A. M. Error correcting codes in quantum theory. Phys. Rev. Lett. 77, 793-797 (1996).

5. Shor, P. W. 37th Symposium on Foundations of Computing 56-65 (IEEE Computer Society Press, Washington DC, 1996).

6. Knill, E. Quantum computing with realistically noisy devices. Nature 434, 39-44 (2005).

7. Raussendorf, R. \& Harrington, J. Fault-tolerant quantum computation with high threshold in two dimensions. Phys. Rev. Lett. 98, 190504 (2007).

8. Reichardt, B. W. Improved ancilla preparation scheme increases fault-tolerant threshold. Preprint at $<$ http://arxiv.org/abs/quant-ph/0406025v1> (2004).

9. Sørensen, A. \& Mølmer, K. Quantum computation with ions in thermal motion. Phys. Rev. Lett. 82, 1971-1974 (1999).

10. Sørensen, A. \& Mølmer, K. Entanglement and quantum computation with ions in thermal motion. Phys. Rev. A 62, 022311 (2000).

11. Sackett, C. A. et al. Experimental entanglement of four particles. Nature 404, 256-259 (2000).

12. Schmidt-Kaler, F. et al. Realization of the Cirac-Zoller controlled-NOT quantum gate. Nature $\mathbf{4 2 2}$, 408-411 (2003)

13. Leibfried, D. et al. Experimental demonstration of a robust, high-fidelity geometric two ion-qubit phase gate. Nature 422, 412-415 (2003).

14. Haljan, P. C. et al. Entanglement of trapped-ion clock states. Phys. Rev. A 72, 062316 (2005).

15. Home, J. P. et al. Deterministic entanglement and tomography of ion spin qubits. New J. Phys. 8, 188 (2006).

16. Riebe, M. et al. Process tomography of ion trap quantum gates. Phys. Rev. Lett. 97, 220407 (2006).

17. Milburn, G. J., Schneider, S. \& James, D. F. V. Ion trap quantum computing with warm ions. Fortschr. Phys. 48, 801-810 (2000).

18. Solano, E., de Matos Filho, R. L. \& Zagury, N. Deterministic Bell states and measurement of the motional state of two trapped ions. Phys. Rev. A 59, R2539-R2543 (1999).

19. Ozeri, R. et al. Errors in trapped-ion quantum gates due to spontaneous photon scattering. Phys. Rev. A 75, 042329 (2007).

20. Roos, C. F. Ion trap quantum gates with amplitude-modulated laser beams. New J. Phys. 10, 013002 (2008).

21. Benhelm, J. et al. Measurement of the hyperfine structure of the $\mathrm{S}_{1 / 2}-\mathrm{D}_{5 / 2}$ transition in ${ }^{43} \mathrm{Ca}^{+}$. Phys. Rev. A 75, 032506 (2007).

22. Roos, C. F., Chwalla, M., Kim, K., Riebe, M. \& Blatt, R. 'Designer atoms' for quantum metrology Nature 443, 316-319 (2006).

23. Mølmer, K. \& Sørensen, A. Multiparticle entanglement of hot trapped ions. Phys. Rev. Lett. 82, 1835-1838 (1999).

24. Leibfried, D. et al. Creation of a six-atom 'Schrödinger cat' state. Nature 438, 639-642 (2005).

25. Chiaverini, J. et al. Realization of quantum error correction. Nature 432, 602-605 (2004).

26. Reichle, R. et al. Experimental purification of two-atom entanglement. Nature 443, 838-841 (2006).

27. Vandersypen, L. M. K. \& Chuang, I. L. NMR techniques for quantum control and computation. Rev. Mod. Phys. 76, 1037-1069 (2004)

28. Häffner, H. et al. Precision measurement and compensation of optical Stark shifts for an ion-trap quantum processor. Phys. Rev. Lett. 90, 143602 (2003).

29. Leibfried, D., Knill, E., Ospelkaus, C. \& Wineland, D. J. Transport quantum logic gates for trapped ions. Phys. Rev. A 76, 032324 (2007).

\section{Acknowledgements}

We gratefully acknowledge the support of the European network SCALA and the Disruptive Technology Office and the Institut für Quanteninformation $\mathrm{GmbH}$. We thank R. Gerritsma and F. Zähringer for help with the experiments.

\section{Author information}

Reprints and permission information is available online at http://npg.nature.com/reprintsandpermissions. Correspondence and requests for materials should be addressed to C.F.R. 